Service social

\title{
Contexte d'autorité et judiciarisation : régression ou redéfinition novatrice de la pratique sociale professionnelle?
}

\section{Liliane Mercier}

Volume 40, numéro 2, 1991

Formation et évolution de la pratique en travail social

URI : https://id.erudit.org/iderudit/706526ar

DOI : https://doi.org/10.7202/706526ar

Aller au sommaire du numéro

Éditeur(s)

École de service social de l'Université Laval

ISSN

1708-1734 (numérique)

Découvrir la revue

Citer cet article

Mercier, L. (1991). Contexte d'autorité et judiciarisation : régression ou redéfinition novatrice de la pratique sociale professionnelle? Service social, 40(2), 43-53. https://doi.org/10.7202/706526ar
Résumé de l'article

Plusieurs analyses sur l'évolution de la profession du service social au Québec associent intervention en contexte d'autorité et déprofessionnali-sation.

L'auteure de cet article nous propose un autre point de vue sur l'état actuel de la pratique sociale.

Elle démontre que les intervenants sociaux exerçant dans les services de protection de la jeunesse au Québec ont non seulement dépassé les contraintes légales et administratives apportées par diverses législations, dont la Loi sur la protection de la jeunesse, mais les ont utilisées pour développer une pratique sociale de qualité. 


\section{Contexte d'autorité et judiciarisation : régression ou redéfinition novatrice de la pratique sociale professionnelle?}

Au cours des dernières années, diverses lois comme la Loi sur la protection de la jeunesse et la Loi sur les jeunes contrevenants et diverses mesures administratives comme celles que préconisait le rapport Harvey ont balisé le changement de l'intervention sociale professionnelle tant sur le plan de la définition du service professionnel que des étapes et des modalités de sa distribution. Entre autres, le formalisme introduit par la judiciarisation, les étapes du processus de distribution du service professionnel ou encore l'émergence d'une intervention professionnelle en contexte d'autorité ont alimenté bon nombre de controverses sur l'autonomie professionnelle effective des travailleurs sociaux et sur la qualité des résultats attendus d'une relation professionnel-client non souhaitée ni sollicitée par celui-ci.

Un relevé des écrits pertinents laisse apparaître une évaluation plutôt négative de ces balises. Plusieurs auteurs associeront celles-ci à des mécanismes de contrôle de l'acte professionnel menant les travailleurs sociaux sur la voie de la " déprofessionnalisation" ". D'autres diront que la relation professionnel-client s'est transformée en mesure de contrôle social des populations à comportements déviants². Bien qu'exactes dans leur ensemble, ces analyses demeurent trop unidimensionnelles. Elles ne rendent pas compte de toute une série de pratiques sociales novatrices qui ont su dépasser les limites et les rigidités imposées par les diverses mesures légales et administratives mentionnées plus haut.

Fondé sur une connaissance approfondie du système de protection de la jeunesse au Québec et sur une expérience professionnelle dans ce domaine plutôt que sur un recensement de la littérature ou un 
relevé systématique de données, ce bref article fait d'abord état de nos réflexions et tente de tracer une esquisse des directions prises par une pratique professionnelle qui n'a pas eu le choix de se transformer au cours des douze dernières années.

Certaines pratiques sociales, à notre avis novatrices, témoignent de l'effort de préservation de l'intégrité de l'acte professionnel en le redéfinissant pour tenir compte du contexte légal et administratif de cet acte. L'intervention en contexte d'autorité n'a pas éliminé la nécessité d'associer volontairement la personne bénéficiaire du service à la définition du problème et à l'élaboration du plan d'action. Pour plusieurs intervenants, ces exigences ont nécessité la mise au point de modèles et techniques d'intervention plus raffinés de manière à concilier ce qui semblait à prime abord antinomique. Au surplus, ces approches ont dû s'ajuster à différents problèmes sociaux (mauvais traitements physiques, abus sexuels, troubles de comportement sérieux, délinquance, etc.) exigeant le développement d'expertises adaptées. Mais plus encore, et bien que cela puisse sembler plutôt paradoxal, les intervenants sociaux se sont servis de ces exigences légales et administratives pour amenuiser certains effets plus ou moins souhaitables du pouvoir du professionnel sur le client. Ce pouvoir, intimement associé à l'acte professionnel, a d'ailleurs été trop facilement occulté dans les modèles de services professionnels privilégiant le volontariat du client ${ }^{3}$.

Cet article propose un autre point de vue sur la pratique sociale. Peut-être y verra-t-on que la profession du service social se porte mieux qu'elle ne paraît. Pour ce faire, nous nous intéresserons d'abord à la notion d'autorité telle qu'elle est véhiculée dans la relation professionnel-client depuis l'origine de la profession au Québec. Par la suite, nous ferons une brève présentation de certains impacts de l'intervention en contexte d'autorité sur la pratique professionnelle des intervenants sociaux en protection de la jeunesse.

\section{La relation travailleur social - client : une relation de pouvoir en soi qu'a clarifiée l'intervention en contexte d'autorité}

Traditionnellement, les modèles d'intervention professionnelle proposés aux étudiants des écoles de service social ont toujours eu pour effet, dans une certaine mesure, de voiler le rapport d'ascendance et d'autorité intrinsèquement associé à la relation professionnel-client. L'obtention d'un statut professionnel est l'une des principales revendications des travailleurs sociaux au cours de leur 
histoire. Les conséquences objectives de cette reconnaissance professionnelle en matière de rapport d'autorité sur le client $n^{\prime}$ ont cependant jamais été vraiment définies et analysées. Une telle lacune sur le plan analytique prend racine dans les différentes conceptions du pouvoir véhiculées en service social. Ces conceptions découlent d'une idéologie professionnelle humaniste diffusée à l'intérieur des différents modèles $d$ 'intervention utilisés par les travailleurs sociaux.

Ainsi la relation professionnel-client est-elle vue au travers de diverses propositions occultant l'éventuel rapport d'autorité du premier sur le deuxième. Cette relation sera successivement présentée comme personnelle, non directive, égalitaire. Elle s'établit de personne à personne sans référence au statut de l'intervenant, à partir d'une définition des besoins par le client ou en alliance avec l'intervenant. Celui-ci, par ses attitudes, laisse percevoir un rapport d'égal à égal avec le client ${ }^{4}$. II n'agit que comme catalyseur de la démarche du client.

Cette volonté de négation réelle ou apparente du rapport d'autorité dans la relation professionnelle est d'autant plus nette que la pratique des intervenants sociaux professionnels s'inscrit dans une rupture avec l'intervention normative à l'origine de la profession.

La professionnalisation du service social québécois francophone débute avec la création de l'École de service social rattachée à l'Université de Montréal en 1940. Le travail social est un complément des pratiques religieuses. La venue de nouveaux agents professionnels du service social ne fait qu'officialiser, en leur donnant le titre d'actes professionnels, des pratiques déjà existantes ${ }^{5}$. Le caractère scientifique de la profession est évincé, puisque toute forme d'intervention ayant pour objectif le bien-être des malheureux doit être soumise aux impératifs de la morale dictée par l'Église :

Dans les situations confliciuelles, de genre concubinage, le travailleur social ne peut les considérer comme normales, même si les concernés affichent un certain bonheur, et accepter d'aider des gens à persévérer dans cet état ${ }^{6}$.

La formation est technique et spécialisée, le vocabulaire est psychologique, mais la pensée et le discours sont religieux et normatifs. Le pouvoir du travail social est idéologique, mais se traduira très concrètement dans la relation avec le client. Dans une situation de concubinage comme celle citée précédemment, il pourra aller jusqu'à un refus de l'accès à certains services (aide matérielle), quelle que soit l'évaluation objective des besoins.

Au milieu des années 50, nous assistons à une rupture entre les travailleurs sociaux et l'Église. Le rôle du travailleur social est maintenant de fournir à la personne en situation de besoin une aide basée sur 
l'application de techniques universelles'. Cette réorientation conduit les travailleurs sociaux vers l'obtention d'un statut professionnel socialement et légalement reconnu (création de la Corporation des travailleurs sociaux du Québec en 1960). Ils se définissent un champ d'intervention spécifique, soit la famille, et prennent le contrôle sur la définition et les structures d'encadrement de leur pratique professionnelle ${ }^{8}$. Les travailleurs sociaux perçoivent alors leur profession comme " neutre et épurée de toute notion de pouvoir puisque maintenant indépendante des institutions au pouvoir (Église ou État) ». Mais qu'en est-il du vécu du client dans cette nouvelle relation professionnelle ?

Pour atteindre son objectif de meilleur fonctionnement social possible de l'individu ou, un peu plus tard, d'amélioration de la condition sociale de la population (objectif défini dans la Réforme sur les services sociaux et de santé), le travailleur social privilégie comme moyen d'intervention la relation d'aide.

La relation d'aide est basée sur la reconnaissance par le client de la compétence du travailleur social à l'aider à régler ses problèmes. L'autorité professionnelle du travailleur social le place, dès le départ, dans une relation d'autorité avec son client ${ }^{9}$. Ce dernier lui reconnaît un droit d'intervention sur des aspects aussi privés que ses relations personnelles et familiales. Certains professionnels formés en service social et spécialisés dans des approches thérapeutiques iront même jusqu'à intervenir sur la personnalité du client. Bien sûr, l'exercice de ce pouvoir d'intervention part d'une des règles de base de la relation d'aide, soit le consentement du client. Le risque d'abus n'est-il cependant pas aussi important ? Les journaux rapportent régulièrement des cas d'abus sexuels faits par des thérapeutes sur leurs clientes. Dans leurs témoignages, les victimes soutiennent l'impossibilité de s'opposer aux désirs de leur thérapeute à cause de sa très grande influence sur elles. Ces situations ne sont évidemment pas le reflet de la nature des relations généralement vécues entre l'intervenant et le client. Elles nous forcent cependant à reconnaître qu'une certaine forme de pouvoir d'influence ou de persuasion est liée à l'autorité professionnelle. En général, le travailleur social utilise d'abord ce rapport d'influence pour motiver le client à recevoir le service. Il est vrai qu'une autre des règles de la relation d'aide est la motivation initiale du client ${ }^{10}$. Cela ne signifie par ailleurs pas que ce dernier partage la définition du problème proposée par le travailleur social. Par exemple, une demande de placement d'enfant faite par le client peut très bien se concrétiser par une thérapie familiale avec maintien du jeune en milieu familial. Il y a alors distorsion entre la demande initiale du client et le service rendu par le travailleur social. Pour en arriver là, le travailleur social doit I'amener à changer sa définition du problème et accepter une solution autre que celle souhaitée au départ. II doit aider le client à reconnaître 
ses résistances à travailler sa relation avec son enfant et le convaincre de recevoir le traitement. Un tel cheminement suppose l'adoption, par le travailleur social, d'une position d'autorité pour influencer le client et le persuader de la justesse et du bien-fondé de la démarche proposée. Comme le souligne Jean Gosselin, le client volontaire peut se retrouver dans des conditions similaires au client non volontaire dans sa relation avec l'intervenant social lorsque la forme d'aide proposée ne correspond pas à sa perception de ses besoins ou encore lorsqu'il se sent $d^{\prime}$ une certaine façon contraint à adopter une solution qui n'est pas sienne ${ }^{11}$. Le fait que l'intervenant $n^{\prime}$ ait pas conscience de cette position d'autorité l'empêche de saisir cette réalité objective chez le client.

La Loi sur la protection de la jeunesse est venue confirmer, de manière explicite, l'utilisation de l'autorité dans la relation d'aide. La protection des enfants impliqués dans une série de situations de compromission (art. 38.38.1) est à la fois la justification et l'objectif de l'intervention sociale exercée dans un contexte d'autorité. Les intervenants sociaux qui sont membres du personnel du Directeur de la protection de la jeunesse (art. 32) sont informés, la plupart du temps par des tiers, de faits pouvant compromettre la sécurité et le développement d'un enfant. Ils ont le pouvoir de statuer sur la nécessité de s'introduire dans le milieu familial de l'enfant pour évaluer la véracité des faits signalés et décider, s'il y a lieu, de l'application de certaines mesures pour protéger l'enfant (art. 32). Ces mesures peuvent être imposées à l'enfant et à ses parents pour une période de vingt-quatre heures (art. 47). Si la mesure doit être prolongée au-delà de ce délai, l'intervenant social doit obtenir le consentement des personnes concernées (enfant s'il est âgé de quatorze ans et plus et parents) à son application (art. 52). Dans le cas où le consentement s'avère impossible à obtenir et où la mesure comporte un hébergement, l'intervenant doit en référer à la Chambre de la jeunesse et demander le prolongement de la mesure déjà appliquée pour une période maximale de trente jours (art. 79). À la fin de la mesure provisoire, le juge entendra les parties et déterminera la mesure jugée la meilleure pour l'enfant et pour ses parents. Par la suite, le pouvoir de l'intervenant social consistera à exécuter l'ordonnance et à surveiller le respect de son contenu par les parties concernées.

Les lignes qui précèdent illustrent, de manière plus concrète, les positions respectives de l'intervenant et du client dans une intervention en contexte d'autorité. Le client n'est pas à l'origine de la relation comme dans le cadre d'une relation d'aide traditionnelle. II ne consent pas, du moins au départ, à l'intervention du professionnel et ne lui reconnaît donc pas la compétence de l'aider à régler ses problèmes. Ce refus du client $n^{\prime}$ annule cependant pas le pouvoir du professionnel, puisque celui-ci a la légitimité sociale et le pouvoir légal d'agir contre sa 
volonté. Mais son pouvoir est un pouvoir de correction plus que d'intervention. II peut imposer au client des mesures qui lui soient extérieures. II ne peut cependant obliger le changement qui, lui, fait référence à une intervention sur la dynamique personnelle, familiale ou sociale. De plus, ce pouvoir de correction doit s'inscrire à l'intérieur de règles légales et administratives clairement établies. Ces règles viennent notamment définir les étapes de l'intervention et des conditions d'application des mesures de protection. Elles prévoient, entre autres, la possibilité d'un refus ou de la contestation par le client de l'autorité de l'intervenant en termes de définition des problèmes et de solutions correctrices de la situation de protection. Cette opposition à l'autorité de l'intervenant provoque la plupart du temps une nouvelle dynamique dans la relation professionnel-client : elle oblige un examen de l'intervention psychosociale par l'instance judiciaire composée de juges et d'avocats. Cette référence au système judiciaire rétablit d'une certaine façon l'équilibre des forces dans le rapport d'autorité. Devant la Chambre de la jeunesse, client et personne autorisée par le Directeur de la protection de la jeunesse sont considérés comme des "parties égales » ayant le droit d'être entendues, de faire valoir leurs points de vue respectifs et d'être représentées par des avocats. Le juge ne se prononcera sur les mesures de protection à appliquer qu'après avoir pris connaissance des éléments de preuve et des positions de chacune des parties. Pourrait-on supposer ici que la judiciarisation permet le passage d'une relation d'autorité à une relation d'égalité entre le professionnel et le client ?

Une réponse affirmative ne tiendrait pas compte de l'existence de rapports sociaux et donc de la place respective qu'occupent généralement l'intervenant et le client dans la société. Un tel questionnement a néanmoins pour intérêt de relativiser l'importance du pouvoir de l'intervenant en contexte d'autorité. Il ouvre aussi une perspective nouvelle sur le défi professionnel que représente l'intervention en contexte d'autorité. Car, si l'intervention en contexte d'autorité associée à la judiciarisation suscite une autre dynamique dans la relation professionnel-client, il serait faux de prétendre que les exigences liées à l'évaluation psychosociale, à l'élaboration des plans d'intervention ou à la nécessité d'associer le client à la réalisation du plan de services s'en trouvent amoindries. Au contraire, elles se déploient dans un réseau de contingences nouvelles, forçant une approche professionnelle modifiée qui va bien au-delà des réalités administratives qui l'encadrent. Dans la prochaine section, nous indiquerons quelques voies qu'a dû emprunter la pratique sociale depuis l'adoption de la Loi sur la protection de la jeunesse en 1979. 


\section{Le défi professionnel de l'intervention en contexte d'autorité et dans un faisceau élargi de contraintes administratives}

"L'intervention en contexte d'autorité est irréconciliable avec toute forme de relation d'aide ". Cette affirmation a souvent été entendue dans le réseau des affaires sociales au cours des douze dernières années. Cela signifie-t-il que tous les intervenants en protection de la jeunesse ont abandonné leur rôle d'aidant pour se satisfaire d'un simple rôle de police sociale ? Si tel était le cas, le débat sur la coexistence de ces deux rôles serait depuis longtemps terminé. La réalité est tout autre. L'objectif de l'intervenant en protection est de mettre fin à une situation où la sécurité ou le développement d'un enfant est compromis. II doit aussi prévenir la répétition de cette situation. Comme la responsabilité de pourvoir aux soins, à l'entretien et à l'éducation de l'enfant revient d'abord aux parents, cela suppose que l'intervenant a pour mandat d'amener le parent à cheminer dans son rôle parental pour non seulement mettre un terme à la situation de protection, mais aussi éviter qu'elle ne se reproduise. Pour atteindre un tel objectif, l'intervenant devra développer des moyens d'intervention qui dépassent la simple mise en place de mécanismes de contrôle des agissement parentaux.

Les situations signalées à la Direction de la protection de la jeunesse sont exceptionnelles au sens de la Loi et nécessitent l'utilisation de moyens extraordinaires d'intervention. Les clientèles trouvées dans ces situations n'utilisent pas les services réguliers (ex. : CLSC) à cause de leur très grande marginalité. Ou encore, elles sont signalées à la Protection de la jeunesse après qu'une série d'interventions ont été réalisées par des aidants naturels ou professionnels œuvrant à partir de la collaboration du client. Ces interventions s'avérant inefficaces ou insuffisantes pour éviter à l'enfant de vivre une situation de compromission ou tout simplement le client décidant d'y mettre un terme, le signalement à la Protection de la jeunesse est alors devenu nécessaire et même obligatoire (art. 39). Il s'agit donc de clientèles très souvent extrêmement démunies tant sur le plan affectif que sur les plans économique et social.

De plus, la Loi sur la protection de la jeunesse a révélé au grand jour une série de problèmes sociaux jusque-là ignorés par la société. Ces problèmes étaient aussi peu connus de l'ensemble des professionnels de la relation d'aide. Les travailleurs sociaux ont dû mettre à jour et souvent développer des connaissances à la fois sur les caractéristiques des situations de protection et des clientèles qui les vivent. Habitués à travailler à partir de plans d'intervention définis à l'avance, les interve- 
nants ont dû devenir habiles à travailler en situation de crise dans des contextes de violence où les éléments de la situation sont complexes et changent rapidement ${ }^{12}$. En même temps, ils ont dû s'adapter aux caractéristiques spécifiques de la relation intervenant-client en contexte d'autorité : il s'agit d'une relation qui présente plusieurs facettes en même temps. "Essentiellement, cette relation est une relation d'affaire, une relation d'autorité, une relation de contrôle, une relation artificielle, une relation $d^{\prime}$ aide $^{13} »$. Non seulement a-t-il fallu se familiariser et développer des habiletés à intervenir selon ces différents types de relation, mais il y a eu aussi nécessité d'apprendre à quel moment et avec quel type de clientèle il y a lieu d'établir un type de relation plutôt qu'un autre. Les stratégies d'intervention sont donc extrêmement importantes en protection de la jeunesse et très différentes de celles traditionnellement connues en relation d'aide en contexte de volontariat.

Rappelons-nous que l'intervenant social agit auprès de personnes qui n'ont pas reconnu de problèmes et souvent refusent de le faire, $n^{\prime}$ ont pas demandé $d^{\prime}$ aide et $n^{\prime}$ ont surtout pas reconnu la compétence de l'intervenant à les aider dans leurs situations de vie. Celui-ci doit alors élaborer des stratégies d'intervention différentes selon l'urgence d'intervenir, la gravité de la situation de protection et les caractéristiques du client. La stratégie de l'intervenant n'est pas la même dans un cas de mauvais traitements très sévères que dans un cas de négligence légère. L'attitude du client (parent ou enfant) oriente aussi le choix de la stratégie d'intervention. Par exemple, la présence d'un parent abusif très violent peut justifier un retrait immédiat de l'enfant, alors que l'enfant peut être laissé dans son milieu si le mauvais traitement est la conséquence d'une perte de contrôle circonstancielle du parent. Le choix de la stratégie est déterminant pour la sécurité de l'enfant et la possibilité d'obtenir l'adhésion du parent à la réalisation du plan d'intervention.

L'intervention en contexte d'autorité ne signifie donc pas l'application mécanique de simples techniques d'intervention. Elle exige l'élaboration de programmes d'intervention adaptés à chaque problème traité. L'intervention auprès de familles négligentes commandera des connaissances et des méthodes d'intervention tout autres que l'intervention auprès de familles vivant l'inceste. Encore là, les expériences professionnelles de traitement étant encore peu nombreuses dans le domaine de la protection de la jeunesse, ce sont les intervenants qui ont souvent eu la responsabilité de les créer ou à tout le moins de les adapter au contexte québécois.

Un autre effet de la Loi sur la protection de la jeunesse est la diversification des rôles de l'intervenant social. La connaissance du système judiciaire, de ses exigences et de ses règles fait maintenant 
partie des apprentissages de l'intervenant. Le statut de "partie » au dossier comporte un grand nombre de désagréments pour l'intervenant qui doit confier à son avocat la conduite judiciaire du dossier. Par contre, le fait de devoir agir à titre de témoin le force à baser son orientation à la fois sur une connaissance détaillée des faits ayant conduit à une situation de compromission et sur une analyse clinique de la vulnérabilité de l'enfant et des capacités parentales de ses parents. L'évaluation psychosociale prend ici toute son importance, puisqu'elle s'appuie non seulement sur une vérification de la matérialité des faits, mais sur une analyse clinique et dynamique des caractéristiques du vécu et de la personnalité de l'enfant et de ses parents. Pour être efficace dans ce nouveau rôle et atteindre une crédibilité aussi grande que celle du témoin-expert, l'intervenant social doit avoir non seulement acquis une série de connaissances cliniques, mais il doit être capable de les systématiser et de les appliquer à chaque cas en tenant compte de ses caractéristiques propres.

Nous ne saurions enfin passer sous silence les conditions parfois très particulières de violence dans lesquelles interviennent les travailleurs sociaux en protection de la jeunesse. Le fait de s'immiscer dans la vie privée des gens sans leur consentement initial peut provoquer des réactions émotives très fortes. Ce non-volontariat prend parfois des formes menaçantes pour l'intervenant. Les menaces verbales et même les agressions physiques font partie du vécu possible de l'intervenant social. Ce dernier, qui agit fréquemment seul et au domicile du client, n'a souvent comme seul moyen de défense que sa capacité à faire tomber les résistances du client pour ensuite récupérer cette agressivité pour mobiliser le parent à modifier ses comportements envers l'enfant. Ce moyen n'est cependant pas toujours efficace et l'intervenant doit alors recourir à des instruments plus coercitifs pour mener à terme son mandat de protection de l'enfant. II y a là un défi que l'intervenant doit relever : celui de ne pas confondre une réaction de violence provoquée par l'intervention avec un comportement violent pouvant compromettre la sécurité ou le développement de l'enfant. Ici encore, une évaluation adéquate de la dangerosité des parents nécessite une bonne connaissance des caractéristiques et du fonctionnement des parents abusifs, une maturité professionnelle et une sécurité personnelle permettant de garder une distance critique vis-à-vis de l'événement de violence vécu.

\section{Conclusion}

Les éléments qui précèdent mettent en évidence quelques-unes des modifications apportées à la pratique sociale pour répondre à ses 
nouvelles exigences légales et administratives. L'obligation formelle d'intervenir en contexte d'autorité a forcé les intervenants sociaux à reconnaître l'existence d'un rapport social différencié entre eux et le client. Reconnaître qu'ils occupent une position dominante dans ce rapport social a mené les intervenants sociaux à définir leurs références idéologiques et à distinguer entre l'imposition d'une norme sociale et la création de modèles d'intervention spécialisés pour faire respecter cette norme. Cette redéfinition s'est faite dans le souci de conserver certaines valeurs professionnelles telles que le respect du client et de son cheminement. Elle a mené à l'élaboration de modèles et de techniques d'intervention dont l'objectif est d'abord de mettre un terme à une situation de compromission pour l'enfant, mais aussi de provoquer un changement des attitudes parentales. La multitude des situations de compromission, de même que la diversité et la complexité des besoins des clientèles des services de protection de la jeunesse, a nécessité le développement d'expertises adaptées à chaque problème social traité et à chaque étape du processus d'intervention. Par exemple, la réception et l'évaluation d'un signalement dépassent la simple collecte de données et exigent de l'intervenant social une capacité à procéder à une évaluation psychosociale complète. De plus, il existe actuellement dans plusieurs Centres de services sociaux du Québec des programmes pour venir en aide à des familles dont les enfants vivent des problèmes tels que l'inceste, la négligence ou encore manifestent des troubles de comportement sérieux. Les intervenants sociaux ont non seulement réussi à sauvegarder la qualité de leur pratique professionnelle mais ils ont utilisé les exigences nouvelles apportées par la Loi sur la protection de la jeunesse pour améliorer et tenter de rétablir un certain équilibre entre le pouvoir du professionnel et celui du client.

Nous terminons en insistant sur la nécessité pour les chercheurs de recenser et de systématiser ces pratiques novatrices. Nous voudrions aussi souligner l'importance d'adapter la formation en service social de manière que les enseignements évitent la " technicité " légale ou administrative comme seul cadre de référence de la pratique professionnelle actuellement en situation d'autorité dans les organismes de services sociaux. En effet, la détérioration accélérée des conditions d'actualisation de la pratique sociale vient, à notre avis, remettre en question le maintien, dans ces organismes, des programmes et expertises récemment développés. Leur transmission aux étudiants inscrits dans les maisons d'enseignement en service social nous semble donc la voie à privilégier pour en assurer la continuité. 


\section{Notes}

${ }^{1}$ G. Renaud, L'éclatement de la profession en Service social, Montréal, Éd. coop. A. St-Martin, 1978, 163 pages.

${ }^{2} \mathrm{~F}$. Lesemann, Du pain et des services. La réforme de la santé et des services sociaux au Québec, Montréal, Éd. coop. A. St-Martin, 1981, 330 pages.

${ }^{3} \mathrm{P}$. Racine, "La Loi de la protection de la jeunesse : son impact sur les professionnels ", Intervention, n 84 , novembre 1989.

4 Ibid., p. 10-12.

${ }^{5}$ G. Renaud, op. cit., p. 26.

${ }^{6}$ R. Raymond, "Service social et catholicisme ", Service social, vol. 7, n 2, été 1957, p. 60-61.

${ }^{7}$ G. Renaud, op. cit., p. 26.

8 lbid., p. 61.

${ }^{9}$ Marie-Claude Esprimont, "Quelques réflexions sur l'intervention en contexte d'autorité ", Intervention, $\mathrm{n}^{\circ} 52$, été 1978, p. 50.

10 Ibid., p. 49.

11 Jean Gosselin, "La relation d'aide en contexte d'autorité ", Intervention, $\mathrm{n}^{\circ} 54$, hiver 1979, p. 6.

${ }^{12}$ Robert Dubé et Marjolaine St-Jules, Protection de l'enfance, réalité de l'intervention, Montréal, Gaëtan Morin Éditeur, 1987, p. 128-133.

${ }^{13}$ Ibid. 